\title{
SARS-CoV-2 cell tropism and multiorgan infection
}

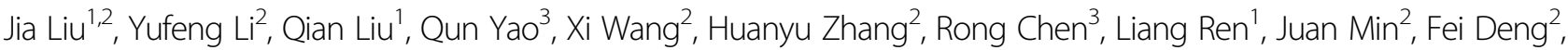 \\ Bing Yan², Liang Liu', Zhihong Hu $\mathbb{E}^{2}$, Manli Wang $\mathbb{E}^{2}$ and Yiwu Zhou ${ }^{1}$
}

\section{Dear Editor,}

To date, the number of confirmed coronavirus disease 2019 (COVID-19) cases has surpassed 100 million, with deaths exceeding 2 million, yet the mechanism by which severe acute respiratory syndrome coronavirus (SARS$\mathrm{CoV})-2$ attacks the body remains unclear. Although SARS-CoV-2 is known to primarily target the lung, it is also believed to cause multi-organ dysfunction and comprehensive studies on SARS-CoV-2 cell tropism in humans are lacking. SARS-CoV-2 exploits the host angiotensin-converting enzyme 2 (ACE2) as its receptor for cell entry ${ }^{1}$, but the correlation between SARS-CoV-2 organ/cell tropism and ACE2 distribution is unclear. Here, we studied these issues via a systemic analysis of postmortem specimens from a 66-year-old female COVID-19 patient who had rapidly developed multiorgan failure. The patient died in the hospital on Day 13 of admission (Day 16 of illness) and her autopsy was performed at $8 \mathrm{~h}$ after death.

To elucidate SARS-CoV-2 tissue tropism, we used immunohistochemical and immunofluorescence staining. Results showed that viral antigens (spike proteins) were highly expressed in pneumocytes and hyperplastic cells around the bronchioles (Supplementary Fig. S1a-c); mucosal epithelia, submucosal glands, and gland ducts of the trachea (Supplementary Fig. S1d-f); mucosal epithelia and glands of the small intestine (Supplementary Fig. S1g, i); distal tubules and collecting ducts of the kidneys (Supplementary Fig. S1j-1); islets of Langerhans, glands, and intraislet ducts of the pancreas (Supplementary Fig. S1m, n); and

\footnotetext{
Correspondence: Zhihong Hu (huzh@wh.iov.cn) or

Manli Wang (wangml@wh.iov.cn) or Yiwu Zhou (zhouyiwu@hust.edu.cn)

'Department of Forensic Medicine, Tongji Medical College of Huazhong University of Science and Technology, Wuhan, Hubei 430010, China

${ }^{2}$ State Key Laboratory of Virology, Wuhan Institute of Virology, Center for Biosafety Mega-Science, Chinese Academy of Sciences, Wuhan, Hubei 430071, China

Full list of author information is available at the end of the article

These authors contributed equally: Jia Liu, Yufeng Li, Qian Liu, Qun Yao.
}

vascular tissues of the brain (Supplementary Fig. S1o, p) and heart (Supplementary Fig. S1q, r). In contrast, few viral antigens were present in the large intestine (Supplementary Fig. S1h) and renal proximal tubules (Supplementary Fig. S1j), and none in the liver (Supplementary Fig. S1s, t). Collectively, these data demonstrate direct multiorgan invasion by, or exposure to, SARS-CoV-2.

To determine the relationship between SARS-CoV-2 organotropism and receptor ACE2 distribution, we performed a co-localization analysis. Co-expression of ACE2 and viral antigen was observed in the lung, trachea, small intestine, kidney, pancreas and heart (Fig. 1a; Supplementary Fig. S2a-i, ii). In the brain, ACE2-expressing cells were detected, but they did not appear to be viral antigenpositive (Supplementary Fig. S2a-iii). In contrast, ACE2 was not expressed in the liver (Supplementary Fig. S2a-iv). These findings suggest that SARS-CoV-2 largely exploits ACE2 as receptor for cell entry in multiple organs.

To elucidate the details of SARS-CoV-2 cell tropism in different tissues, we analyzed the co-localization of viral antigens with various cell lineage markers by using multiplex immunofluorescence assay. In the lung, viral antigens were detected in major epithelial cell types of the bronchi/ bronchioles including keratin 5-positive $\left(\mathrm{KRT}^{+}\right)$basal, acetylated $\alpha$-tubulin antibody-positive $\left(\mathrm{Ac}^{\left.-\mathrm{Tub}^{+}\right)}\right.$ciliated, and club cell 10-positive $\left(\mathrm{CC}^{+} 0^{+}\right)$club cells (Fig. 1b-i-iv). Although we found that some goblet marker mucin $5 \mathrm{~A}$ (MUC5A)-positive cells were infected (Fig. 1b-v), these cells also co-expressed $\mathrm{CC} 10$ (Fig. 1b-iv), suggesting they might be transdifferentiated/differentiated from club cells. The virus also infected a few receptor for advanced glycation end products-positive $\left(\mathrm{RAGE}^{+}\right)$alveolar type (AT) 1 cells as well as surfactant protein C-positive $\left(\mathrm{SFTPC}^{+}\right)$AT2 cells (Fig. 1b-vi-viii). Some viral positive cells were found to co-express both AT1 and AT2 cell markers, but were morphologically distinct from the flat mature AT1 cells (Supplementary Fig. S2b-i, ii), suggesting the infected AT1 cells might be transdifferentiated/differentiated from 


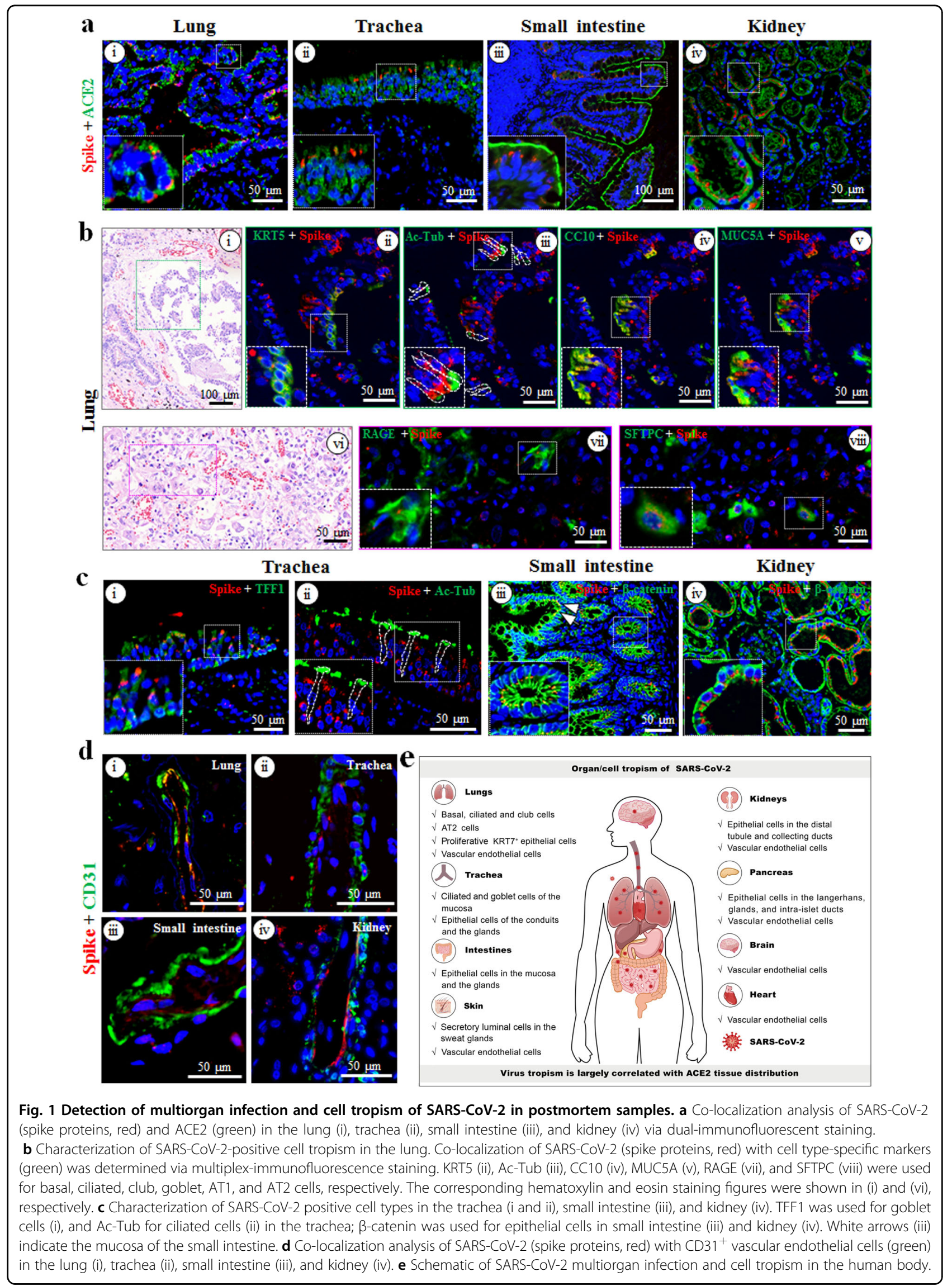


AT2 cells. Interestingly, viral antigen signals were detected at high levels in many proliferative $\mathrm{KRT}^{+}$epithelial cells (Supplementary Fig. S2c-i) and in a few $\mathrm{Ki} 67^{+}$proliferative cells (Supplementary Fig. S2c-ii). Collectively, our results suggest that SARS-CoV-2 mainly infected basal, ciliated, club, AT2, and proliferative $\mathrm{KRT}^{+}$epithelial cells in the lung.

In the trachea, epithelial marker KRT7 clearly revealed viral infection in epithelial cells of the mucosa (Supplementary Fig. S3a-i), conduits, and glands (Supplementary Fig. S3a-ii). Among mucosal epithelial cells, viral antigens were found in trefoil factor 1-positive $\left(\mathrm{TFF} 1^{+}\right.$) goblet (Fig. 1c-i) and Ac-Tub ${ }^{+}$ciliated cells (Fig. 1c-ii), but few were found in $\mathrm{KRT}^{+}$basal (Supplementary Fig. S3a-iii) and $\mathrm{CC} 0^{+}$club (Supplementary Fig. S3a-iv) cells. In the small intestines, viral antigens were abundant in mucosal (white arrows) and glandular (white box) $\beta$-catenin ${ }^{+}$epithelial cells (Fig. 1c-iii). In the kidneys, $\beta$-catenin ${ }^{+}$-distal tubule (Fig. 1c-iv) and collecting duct (Supplementary Fig. S3b-i) epithelial cells were highly sensitive to SARS-CoV-2 infection. $\mathrm{CD} 1^{+}$glomerular endothelial cells also showed positive signals for SARS-CoV-2 (Supplementary Fig. S3b-ii), but viral antigens were not detected in the proximal tubules (Supplementary Fig. S3b-iii).

The virus infected the $\mathrm{CD} 31^{+}$vascular endothelial cells in the lung, trachea, small intestine, and kidney (Fig. 1d) as well as the pancreas, heart and brain (Supplementary Fig. S4a). As expected, ACE2 was observed in the vascular endothelial cells of different tissues (Supplementary Fig. S4b). Taken together, these results confirm that SARS-CoV-2 preferentially targets vascular endothelial cells in multiple organs.

It is known that the respiratory system is a major target of SARS-CoV-2. Recent studies showed that SARS-CoV-2 targets ciliated and AT2 cells in airway and alveolar regions ${ }^{2}$, and consistently epithelium ciliated cells and AT2 in lung are the major cell types that co-express ACE2 and co-receptor transmembrane protease seine 2 $\left(\right.$ TMPRSS2) ${ }^{3}$. Our study identified viral infection in basal-, ciliated-, club-, and AT2 cells in the lungs (Fig. 1b) and in epithelial goblet and ciliated cells of the trachea (Fig. 1c-i, ii). Proliferative hyperplastic cells in the lungs were among the major target cells (Supplementary Fig. S2c), which is in line with a recent report that identified newly proliferated cells in the respiratory epithelia of COVID-19 patients ${ }^{4}$. It is likely that those cells were proliferated upon viral infection, or they were generated to repair lung damage but were hijacked for virus amplification.

Expressions of ACE2 and TMPRSS2 in the lungs and trachea were further analyzed. In the lung, ACE2 and TMPRSS2 were easily detected in some ciliated-, club- and goblet cells, and many of them were viral-positive (Supplementary Fig. S5b-d). However, ACE2 and TMPRSS2 were rarely detected in the basal cells where virus infection virtually occurred (Supplementary Fig. S5a), suggesting the entry factors by which the virus exploits in basal cells need further investigation. Co-expression of ACE2 and TMPRSS2 was identified in some AT2 cells and consistently, viral infection was detected (Supplementary Fig. S5f). By contrast, ACE2 and TMPRSS2 were rarely detected in AT1 cells (Supplementary Fig. S5e), which may explain why SARSCoV-2 was hardly detected in AT1 cells. The proliferative $\mathrm{KRT7}^{+}$cells co-expressed high levels of ACE2 and TMRPSS2, supporting the finding that these cells were permissive for virus infection (Supplementary Figs. S5g and S2c-i). In trachea, ACE2 and TMPRSS2 could be easily detected in ciliated-, and goblet cells, and consistently, many of them were viral positive (Supplementary Fig. S6a, b). Therefore, except for the basal cells in the lung, our results generally supported the correlation between ACE2/ TMPRSS2 distribution and SARS-CoV-2 cell tropism in the lung and trachea. The discrepancies between our results and other studies ${ }^{2,3}$, may be due to different methodologies and different human samples used. For example, ACE2 expression appeared to be induced by interferon or dysregulated in patients with pre-existing pulmonary diseases ${ }^{2,3}$.

Accumulating evidence suggests that the intestines might be a target organ ${ }^{5}$ and gastrointestinal symptoms have been reported in some COVID-19 patients. Here, high ACE2 expression levels and an abundance of viral antigens were consistently detected in enterocytes of the small intestine (Fig. 1a-iii), which concurs with a report that ACE2 expression was high in the microvilli of intestines ${ }^{6}$. Our findings are also supported by studies showing successful isolation of SARS-CoV-2 from the stool samples of patients with severe COVID-19 ${ }^{7}$. Collectively, the evidence implies that the gastrointestinal system might serve as a SARS-CoV-2 transmission route and amplification factory.

Acute kidney injury has been reported in up to $25 \%$ of critically-ill COVID-19 patients $^{8}$, and SARS-CoV-2 has been isolated from the urine samples of some patients ${ }^{9}$. We observed high ACE2 expression in the proximal, distal tubules, and collecting ducts (Fig. 1a-iv); however, viral antigens were only identified in the distal tubules and collecting ducts, not in the proximal tubules (Fig. 1c-iv; Supplementary Figs. S1j-l and S3b-iii). This distribution pattern is similar to that of SARS-CoV ${ }^{10}$, but is different from some studies showing SARS-CoV-2-infected proximal tubules ${ }^{11}$. This suggests that in addition to ACE2, other host factors, such as co-receptors, immune response and viral transmission, may also play critical roles for virus infection in these tissues. It remains unknown whether the viral distribution we observed is case specific. Nevertheless, our data and those in other reports suggest that acute kidney injury is a pathological consequence of direct viral infection and that urine is a possible SARSCoV-2 transmission route. 
Vascular endothelial injury, endotheliitis, and coagulation dysfunction have been reported in some COVID-19 patients; such conditions are likely associated with disease severity ${ }^{12}$. Actually, direct SARS-CoV-2 infection of lung microvascular endothelial cells in human and cultured blood vessel organoids has been reported ${ }^{12-14}$. We detected SARS-CoV-2 infection in the $\mathrm{CD} 31^{+}$vascular endothelial cells of multiple organs (Fig. 1d; Supplementary Fig. S4a). ACE2 has been reported in arterial and venous endothelial cells; ${ }^{14}$ likewise, we detected ACE2 expression in the vascular endothelial cells of multiple organs (Supplementary Fig. S4b). The fact that SARSCoV-2 infection in the vascular endothelial cells of various organs induces both direct and indirect damage (e.g., coagulation dysfunction) suggests that blood vessels are important target tissues that might represent a dissemination route within the human body.

In conclusion, our results identified SARS-CoV-2 cell tropism in multiple organs (Fig. 1e), indicating that SARSCoV-2 infects not only the respiratory system (e.g., lungs and trachea) but also the kidneys, small intestines, pancreas, blood vessels, and other tissues. Recently, we disclosed that SARS-CoV-2 also targeted sweat glands and vascular endothelial cells in the skin ${ }^{15}$. These findings suggest that direct viral infection could, at least partially, contribute to multiorgan injury. Our results also proved a possible correlation between SARS-CoV-2 organotropism and ACE2 distribution, providing supporting evidence for the multiorgan infection of the virus. While our study was limited by its size, its sheds new light into the mechanism of viral infection/transmission and provides valuable information for COVID-19 control.

\section{Acknowledgements}

We acknowledge Mingyue Xu, Hengrui Hu, Xijia Liu, Zhengyuan Su, Min Zhou for their critical support. We thank Jia Wu, Hao Tang and Jun Liu from National Biosafety Laboratory (Wuhan), Chinese Academy of Sciences for their support during the study. The study was supported in part by grants from Ministry of Science and Technology of China (2020YFC0844700 and 2020FYC0841700), Hubei Science and Technology funding (2020FCA003 and 2020FCA045), Open Research Fund Program of the State Key Laboratory of Virology of China (2021lOV004), and the National Natural Science Foundation of China (31621061).

\section{Author details}

'Department of Forensic Medicine, Tongji Medical College of Huazhong University of Science and Technology, Wuhan, Hubei 430010, China. ${ }^{2}$ State Key Laboratory of Virology, Wuhan Institute of Virology, Center for Biosafety MegaScience, Chinese Academy of Sciences, Wuhan, Hubei 430071, China. ${ }^{3}$ Jinyintan Hospital, Wuhan, Hubei 430023, China

\section{Author contributions}

J.L., M.W., Z.H. and Y.Z. designed and coordinated the study, analyzed and interpreted the results. L.L., Y.Z., Q.L., Q.Y., R.C., L.R. and F.D. contributed to performance of autopsies and tissue collection. J.L., Y.L., X.W., H.Z., J.M. and B.Y participated in multiple experiments. M.W., Z.H., J.L. and Y.Z. wrote the manuscript. M.W., Z.H. and Y.Z. approved the final manuscript.

\section{Conflict of interest}

The authors declare that they have no conflict of interest.

\section{Publisher's note}

Springer Nature remains neutral with regard to jurisdictional claims in published maps and institutional affiliations.

Supplementary information The online version contains supplementary material available at https://doi.org/10.1038/s41421-021-00249-2.

Received: 15 October 2020 Accepted: 8 February 2021

Published online: 23 March 2021

References

1. Zhou, P. et al. A pneumonia outbreak associated with a new coronavirus of probable bat origin. Nature $\mathbf{5 7 9}, \mathbf{2 7 0 - 2 7 3 ~ ( 2 0 2 0 ) . ~}$

2. Hou, Y. J. et al. SARS-CoV-2 reverse genetics reveals a variable infection gradient in the respiratory tract. Cell 182, 429-446.e14 (2020).

3. Ziegler, C. G. K. et al. SARS-CoV-2 receptor ACE2 is an interferon-stimulated gene in human airway epithelial cells and is detected in specific cell subsets across tissues. Cell 181, 1016-1035 e1019 (2020).

4. Fang, Y. et al. Distinct stem/progenitor cells proliferate to regenerate the trachea, intrapulmonary airways and alveoli in COVID-19 patients. Cell Res. 30, 705-707 (2020).

5. Xiao, F. et al. Evidence for Gastrointestinal Infection of SARS-CoV-2. Gastroenterology 158, 1831-1833.e3 (2020).

6. Hikmet, F., Mear, L., Uhlen, M. \& Lindskog, C. The protein expression profile of ACE2 in human tissues. Mol. Syst. Biol. 16, e9610 (2020).

7. Xiao, F. et al. Infectious SARS-CoV-2 in feces of patient with severe COVID-19. Emerg. Infect. Dis. 26, 1920-1922 (2020).

8. Gabarre, P. et al. Acute kidney injury in critically ill patients with COVID-19. Intensive Care Med. 46, 1339-1348 (2020).

9. Sun, J. et al. Isolation of infectious SARS-CoV-2 from urine of a COVID-19 patient. Emerg. Microbes Infect. 9, 991-993 (2020).

10. Ding, Y. et al. Organ distribution of severe acute respiratory syndrome (SARS) associated coronavirus (SARS-CoV) in SARS patients: implications for pathogenesis and virus transmission pathways. J. Pathol. 203, 622-630 (2004).

11. Menter, T. et al. Post-mortem examination of COVID19 patients reveals diffuse alveolar damage with severe capillary congestion and variegated findings of lungs and other organs suggesting vascular dysfunction. Histopathology 77, 198-209 (2020).

12. Ackermann, M. et al. Pulmonary vascular endothelialitis, thrombosis, and angiogenesis in Covid-19. N. Engl. J. Med. 383, 120-128 (2020).

13. Varga, Z. et al. Endothelial cell infection and endotheliitis in COVID-19. Lancet 395, 1417-1418 (2020).

14. Monteil, V. et al. Inhibition of SARS-CoV-2 infections in engineered human tissues using clinical-grade soluble human ACE2. Cell 181, 905-913 (2020).

15. Liu, J. et al. Infection of human sweat glands by SARS-CoV-2. Cell Discov. 6, 84 (2020). 